\title{
Commutative Algebra of $n$ Points in the Plane
}

\author{
MARK HAIMAN \\ WITH AN APPENDIX BY EZRA MILLER
}

\begin{abstract}
We study questions arising from the geometry of configurations of $n$ points in the affine plane $\mathbb{C}^{2}$. We first examine the ideal of the locus where some two of the points coincide, and then study the rings of invariants and coinvariants for the action of the symmetric group $S_{n}$ permuting the points among themselves. We also discuss the ideal of relations among the slopes of the lines that connect the $n$ points pairwise, which is the subject of beautiful and surprising results by Jeremy Martin.
\end{abstract}

\section{Contents}

Introduction

Lecture 1: A Subspace Arrangement $\quad 154$

Lecture 2: A Ring of Invariants $\quad 161$

Lecture 3: A Remarkable Gröbner Basis 168

Appendix: Hilbert Schemes of Points in the Plane 172

$\begin{array}{lr}\text { References } & 179\end{array}$

\section{Introduction}

These lectures address commutative algebra questions arising from the geometry of configurations of $n$ points in the affine plane $\mathbb{C}^{2}$. In the first lecture, we study the ideal of the locus where some two of the points coincide. We are led naturally to consider the action of the symmetric group $S_{n}$ permuting the points among themselves. This provides the topic for the second lecture, in which we study the rings of invariants and coinvariants for this action. As you can see, we have chosen to study questions that involve rather simple and naive geometric considerations. For those who have not encountered this subject before, it may come as a surprise that the theorems which give the answers are quite remarkable, and seem to be hard.

One reason for the subtlety of the theorems is that lurking in the background is the more subtle geometry of the Hilbert scheme of points in the plane. The 
special properties of this algebraic variety play a role in the proofs of the theorems. The involvement of the Hilbert scheme in the proofs means that at present the theorems apply only to points in the plane, even though we could equally well raise the same questions for points in $\mathbb{C}^{d}$, and conjecturally we expect them to have similar answers.

In the third lecture, we change perspective slightly, by introducing the $\left(\begin{array}{l}n \\ 2\end{array}\right)$ lines connecting the points in pairs, and asking for the ideal of relations among the slopes of these lines when the points are in general position (that is, no two points coincide). We present a synopsis of the beautiful and surprising results on this problem found by my former student, Jeremy Martin.

\section{Lecture 1: A Subspace Arrangement}

We consider ordered $n$-tuples of points in the plane, denoted by

$$
P_{1}, \ldots, P_{n} \in \mathbb{C}^{2} .
$$

We work over $\mathbb{C}$ to keep things simple and geometrically concrete, although some of the commutative algebra results remain true over more general ground rings. Assigning the points coordinates

$$
x_{1}, y_{1}, \ldots, x_{n}, y_{n}
$$

we identify the space $E$ of all $n$-tuples $\left(P_{1}, \ldots, P_{n}\right)$ with $\mathbb{C}^{2 n}$. The coordinate ring of $E$ is then the polynomial ring

$$
\mathbb{C}[E]=\mathbb{C}[\boldsymbol{x}, \boldsymbol{y}]=\mathbb{C}\left[x_{1}, y_{1}, \ldots, x_{n}, y_{n}\right]
$$

in $2 n$ variables. Let $V_{i j}$ be the locus where $P_{i}=P_{j}$, that is, the codimension-2 subspace of $E$ defined by the equations $x_{i}=x_{j}$ and $y_{i}=y_{j}$. The locus

$$
V=\bigcup_{i<j} V_{i j}
$$

where some two points coincide is a subspace arrangement of $\left(\begin{array}{l}n \\ 2\end{array}\right)$ codimension-2 subspaces in $E$. Evidently, $V$ is the zero locus of the radical ideal

$$
I=I(V)=\bigcap_{i<j}\left(x_{i}-x_{j}, y_{i}-y_{j}\right) .
$$

The central theme of today's lecture is: What does the ideal I look like?

As a warm-up, we consider the much easier case of $n$ points on a line. Then we only have coordinates $x_{1}, \ldots, x_{n}$, and the analog of $I$ is the ideal

$$
J=\bigcap_{i<j}\left(x_{i}-x_{j}\right) \subseteq \mathbb{C}[\boldsymbol{x}] .
$$

This ideal has some easily checked properties. 
(1) $J$ is the principal ideal $(\Delta(\boldsymbol{x}))$ generated by the Vandermonde determinant

$$
\Delta(\boldsymbol{x})=\prod_{i<j}\left(x_{i}-x_{j}\right)=\operatorname{det}\left[\begin{array}{cccc}
1 & x_{1} & \ldots & x_{1}^{n-1} \\
1 & x_{2} & \ldots & x_{2}^{n-1} \\
\vdots & \vdots & & \vdots \\
1 & x_{n} & \ldots & x_{n}^{n-1}
\end{array}\right] .
$$

(2) $J$ is (trivially) a free $\mathbb{C}[\boldsymbol{x}]$ module with generator $\Delta(\boldsymbol{x})$.

(3) $J^{m}=J^{(m)} \stackrel{\text { def }}{=} \bigcap_{i<j}\left(x_{i}-x_{j}\right)^{m}$, that is, the powers of $J$ are equal to its symbolic powers. This is clear, since both ideals are equal to $\left(\Delta(\boldsymbol{x})^{m}\right)$.

(4) The Rees algebra $\mathbb{C}[\boldsymbol{x}][t J]$ is Gorenstein. In fact, it's just a polynomial ring in $n+1$ variables.

All this follows from the fact that $J$ is the ideal of a hyperplane arrangement. In general, one cannot say much about the ideal of an arrangement of subspaces of codimension 2 or more. However, our ideal $I$ is rather special, so let's try to compare its properties with those listed above for $J$.

Beginning with property (1), we can observe that $I$ has certain obvious elements. The symmetric group $S_{n}$ acts on $E$, permuting the points $P_{i}$. In coordinates, this is the diagonal action

$$
\sigma x_{i}=x_{\sigma(i)}, \sigma y_{i}=y_{\sigma(i)} \quad \text { for } \sigma \in S_{n} .
$$

We denote the sign character of $S_{n}$ by

$$
\varepsilon(\sigma)=\left\{\begin{aligned}
1 & \text { if } \sigma \text { is even } \\
-1 & \text { if } \sigma \text { is odd }
\end{aligned}\right.
$$

Let

$$
\mathbb{C}[\boldsymbol{x}, \boldsymbol{y}]^{\varepsilon}=\left\{f \in \mathbb{C}[\boldsymbol{x}, \boldsymbol{y}]: \sigma f=\varepsilon(\sigma) f \text { for all } \sigma \in S_{n}\right\}
$$

be the space of alternating polynomials. Any alternating polynomial $f$ satisfies

$$
\begin{array}{r}
f\left(x_{1}, y_{1}, \ldots, x_{i}, y_{i}, \ldots, x_{j}, y_{j}, \ldots, x_{n}, y_{n}\right) \\
\quad=-f\left(x_{1}, y_{1}, \ldots, x_{j}, y_{j}, \ldots, x_{i}, y_{i}, \ldots, x_{n}, y_{n}\right),
\end{array}
$$

which immediately implies that $f$ vanishes on every $V_{i j}$, that is, $f$ belongs to $I$.

There is a natural vector space basis for $\mathbb{C}[\boldsymbol{x}, \boldsymbol{y}]^{\varepsilon}$. Namely, let $\boldsymbol{x}^{\alpha} \boldsymbol{y}^{\beta}=$ $x_{1}^{\alpha_{1}} y_{1}^{\beta_{1}} \ldots x_{n}^{\alpha_{n}} y_{n}^{\beta_{n}}$ be a monomial, and put

$$
A\left(\boldsymbol{x}^{\alpha} \boldsymbol{y}^{\beta}\right)=\sum_{\sigma \in S_{n}} \varepsilon(\sigma) \sigma\left(\boldsymbol{x}^{\alpha} \boldsymbol{y}^{\beta}\right)
$$

If the exponent pairs $\left(\alpha_{i}, \beta_{i}\right)$ are not all distinct, then $A\left(\boldsymbol{x}^{\alpha} \boldsymbol{y}^{\beta}\right)=0$. If they are all distinct, set $D=\left\{\left(\alpha_{1}, \beta_{1}\right), \ldots,\left(\alpha_{n}, \beta_{n}\right)\right\} \subseteq \mathbb{N} \times \mathbb{N}$. Then $A\left(\boldsymbol{x}^{\alpha} \boldsymbol{y}^{\beta}\right)$ is given by a bivariate analog of the Vandermonde determinant

$$
A\left(\boldsymbol{x}^{\alpha} \boldsymbol{y}^{\beta}\right)=\Delta_{D}=\operatorname{det}\left[\begin{array}{ccc}
x_{1}^{\alpha_{1}} y_{1}^{\beta_{1}} & \ldots & x_{1}^{\alpha_{n}} y_{1}^{\beta_{n}} \\
\vdots & & \vdots \\
x_{n}^{\alpha_{1}} y_{n}^{\beta_{1}} & \ldots & x_{n}^{\alpha_{n}} y_{n}^{\beta_{n}}
\end{array}\right] \text {, }
$$


which only depends on $D$, up to sign. It is easy to see that the set of all such polynomials

$$
\left\{\Delta_{D}: D \subseteq \mathbb{N} \times \mathbb{N},|D|=n\right\}
$$

is a vector space basis of $\mathbb{C}[\boldsymbol{x}, \boldsymbol{y}]^{\varepsilon}$. In particular, the ideal they generate is the same as the ideal generated by all alternating polynomials. We have just seen that this ideal is contained in $I$.

Theorem 1.1. We have $I=\left(\Delta_{D}: D \subseteq \mathbb{N} \times \mathbb{N},|D|=n\right)$.

As far as I know, this is not an easy theorem. We will say something about its proof later on. Before that, we discuss briefly the question of finding a minimal set of generators for $I$, and take up the analogs of the other properties (2)-(4) that we had for $J$.

Note that $I$ is a homogeneous ideal - in fact it is doubly homogeneous, with respect to the double grading given by degrees in the $\boldsymbol{x}$ and $\boldsymbol{y}$ variables separately. It follows that a set of homogeneous generators for $I$, for example a subset of the $\Delta_{D}$ 's, is minimal if and only if its image is a vector space basis of

$$
I /(\boldsymbol{x}, \boldsymbol{y}) I \text {. }
$$

It turns out that we know exactly what the size of such a minimal generating set must be, although no one has yet succeeded in finding an explicit choice of minimal generators.

Theorem 1.2. The dimension of $I /(\boldsymbol{x}, \boldsymbol{y}) I$ is equal to the Catalan number

$$
C_{n}=\frac{1}{n+1}\left(\begin{array}{c}
2 n \\
n
\end{array}\right)
$$

Indeed, quite a bit more can be said. The space $M=I /(\boldsymbol{x}, \boldsymbol{y}) I$ is doubly graded, say $M=\bigoplus_{r, s} M_{r, s}$. Define a " $q, t$-analog" of the Catalan number by

$$
C_{n}(q, t)=\sum_{r, s} t^{r} q^{s} \operatorname{dim} M_{r, s}
$$

According to Theorem 1.2 we then have $C_{n}(1,1)=C_{n}$. From geometric considerations involving the Hilbert scheme we have a formula for $C_{n}(q, t)$ [Haiman 1998; 2002], and Theorem 1.2 is proved by specializing the formula to $q=t=1$. The formula gives $C_{n}(q, t)$ as a complicated rational function of $q, t$ that on its face is not even obviously a polynomial. However, Garsia and Haglund [Garsia and Haglund 2001; 2002] discovered a simple combinatorial interpretation of the formula, as follows. Let $\mathscr{D}$ be the set of integer sequences

$$
\lambda_{1} \geq \lambda_{2} \geq \cdots \geq \lambda_{n-1} \geq 0
$$

satisfying

$$
\lambda_{i} \leq n-i \text { for all } i \text {. }
$$


In other words, $\mathscr{D}$ is the set of partitions whose Young diagram fits inside that of the partition $(n-1, n-2, \ldots, 1)$. It is well-known that the number of these is the Catalan number $C_{n}$. For each $\lambda \in \mathscr{D}$, define

$$
\begin{aligned}
& a(\lambda)=\sum_{i}(n-i-\lambda), \\
& b(\lambda)=\left|\left\{i<j: \lambda_{i}-\lambda_{j}+i-j \in\{0,1\}\right\}\right| .
\end{aligned}
$$

Garsia and Haglund showed that

$$
C_{n}(q, t)=\sum_{\lambda \in \mathscr{D}} q^{a(\lambda)} t^{b(\lambda)} .
$$

Problem 1.3. Find a rule associating to each $\lambda \in \mathscr{D}$ an $n$-element subset $D(\lambda) \subseteq \mathbb{N} \times \mathbb{N}$ in such a way that $\operatorname{deg}_{\boldsymbol{y}} \Delta_{D(\lambda)}=a(\lambda), \operatorname{deg}_{\boldsymbol{x}} \Delta_{D(\lambda)}=b(\lambda)$, and the set $\left\{\Delta_{D(\lambda)}: \lambda \in \mathscr{D}\right\}$ generates $I$.

A solution to this problem would give a new and in some sense improved proof of the Garsia-Haglund result. One can proceed similarly for the powers of $I$, defining

$$
M^{(m)}=I^{m} /(\boldsymbol{x}, \boldsymbol{y}) I^{m}
$$

and

$$
C_{n}^{(m)}(q, t)=\sum_{r, s} t^{r} q^{s} \operatorname{dim} M_{r, s}^{(m)} .
$$

Again there is a formula for $C_{n}^{(m)}(q, t)$ from geometry. There is also a conjectured combinatorial interpretation, as follows. Let $\mathscr{D}^{(m)}$ be the set of integer sequences

$$
\lambda_{1} \geq \lambda_{2} \geq \cdots \geq \lambda_{n-1} \geq 0
$$

satisfying

$$
\lambda_{i} \leq m(n-i) \text { for all } i \text {. }
$$

In other words, we now allow partitions whose Young diagram fits inside that of $m \cdot(n-1, n-2, \ldots, 1)$. For each $\lambda \in \mathscr{D}^{(m)}$, define

$$
\begin{aligned}
a^{(m)}(\lambda) & =\sum_{i}(m(n-i)-\lambda), \\
b^{(m)}(\lambda) & =\left|\left\{i<j: \lambda_{i}-\lambda_{j}+m(i-j) \in\{0,1, \ldots, m\}\right\}\right| .
\end{aligned}
$$

ConjeCture 1.4. We have $C_{n}^{(m)}(q, t)=\sum_{\lambda \in \mathscr{D}(m)} q^{a^{(m)}(\lambda)} t^{b^{(m)}(\lambda)}$.

Problem 1.5. Find generators for $I^{m}$ indexed by elements $\lambda \in \mathscr{D}^{(m)}$, with $\boldsymbol{y}$-degree equal to $a^{(m)}(\lambda)$ and $\boldsymbol{x}$-degree equal to $b^{(m)}(\lambda)$.

It is known that $C_{n}^{(m)}(q, 1)=\sum_{\lambda \in \mathscr{D}(m)} q^{a^{(m)}(\lambda)}$, and hence in particular that $\operatorname{dim} I^{m} /(\boldsymbol{x}, \boldsymbol{y}) I^{m}=C_{n}(1,1)=\left|\mathscr{D}^{(m)}\right|$. The generating set given by a solution to Problem 1.5 would therefore be minimal, so Conjecture 1.4 would follow automatically. 
Now we ask whether $I$ has an analog of property (2) for $J$. It certainly cannot be that $I$ is a free $\mathbb{C}[\boldsymbol{x}, \boldsymbol{y}]$-module, for then $\mathbb{C}[\boldsymbol{x}, \boldsymbol{y}] / I$ would have depth $2 n-1$, whereas it has dimension $2 n-2$. What we have instead is that $I$ is a free module with respect to either set of variables alone.

Theorem 1.6. The ideal I is a free $\mathbb{C}[\boldsymbol{y}]$-module.

This theorem is best possible, modulo one detail. The ideal $I$ has an extra degree of freedom: it is invariant with respect to $\boldsymbol{x}$-translations mapping each $x_{i}$ to $x_{i}+a$. This invariance holds for $I /(\boldsymbol{y}) I$ as well, and implies that $I /(\boldsymbol{y}) I$ is a free $\mathbb{C}\left[x_{1}\right]$-module (say). Hence Theorem 1.6 actually implies that $I$ is a free $\mathbb{C}\left[\boldsymbol{y}, x_{1}\right]$-module, and in particular has depth at least $n+1$. On the other hand, it is easy to see that $\Delta(\boldsymbol{y})$ represents a nonzero element of $I /(\boldsymbol{y}) I$ annihilated by $\left(x_{1}-x_{2}, \ldots, x_{n-1}-x_{n}\right)$. This implies that $\operatorname{depth} I /(\boldsymbol{y}) I \leq 1$ and hence $\operatorname{depth} I=$ $n+1$.

Next we turn to property (3), the coincidence of powers with symbolic powers.

TheOrem 1.7. We have $I^{m}=I^{(m)} \stackrel{\text { def }}{=} \bigcap_{i<j}\left(x_{i}-x_{j}, y_{i}-y_{j}\right)^{(m)}$ for all $m$.

In fact, Theorems 1.1, 1.6, and 1.7 are all plainly corollaries to the following two statements.

Theorem 1.8. For all $m$, the $m$-th power of the ideal $\left(\Delta_{D}: D \subseteq \mathbb{N} \times \mathbb{N},|D|=n\right)$ is a free $\mathbb{C}[\boldsymbol{y}]$-module.

Corollary 1.9. For all $m$, we have $I^{(m)}=\left(\Delta_{D}: D \subseteq \mathbb{N} \times \mathbb{N},|D|=n\right)^{m}$.

On the maxim that every mathematics lecture should contain one proof, we sketch how Theorem 1.8 implies Corollary 1.9. Abbreviating $\left(\Delta_{D}: D \subseteq \mathbb{N} \times\right.$ $\mathbb{N},|D|=n)$ to $\left(\Delta_{D}\right)$, we clearly have

$$
\left(\Delta_{D}\right)^{m} \subseteq I^{(m)} .
$$

Localizing at any point $\mathbf{P} \in E$ with not all $P_{i}$ equal, one shows that both $\left(\Delta_{D}\right)_{\mathbf{P}}$ and $I_{\mathbf{P}}^{(m)}$ factor locally into products of the corresponding ideals in subsets of the variables. On the open set $U$ where some $P_{i} \neq P_{j}$ we can therefore assume locally that $\left(\Delta_{D}\right)_{\mathbf{P}}^{m}=I_{\mathbf{P}}^{(m)}$, by induction on $n$.

Now Theorem 1.8 implies that $\mathbb{C}[\boldsymbol{x}, \boldsymbol{y}] /\left(\Delta_{D}\right)^{m}$ has depth $\geq n-1$ as a $\mathbb{C}[\boldsymbol{y}]$ module. In particular, $\left(\Delta_{D}\right)^{m}$ cannot have an associated prime supported in $V\left(y_{1}-y_{2}, \ldots, y_{n-1}-y_{n}\right)$, if $n \geq 3$. In other words, if $f \in \mathbb{C}[\boldsymbol{x}, \boldsymbol{y}]$ belongs to the localization $\left(\Delta_{D}\right)_{Q}^{m}$ for all $Q \in(\operatorname{Spec} \mathbb{C}[\boldsymbol{y}]) \backslash V\left(y_{1}-y_{2}, \ldots, y_{n-1}-y_{n}\right)$, then $f \in\left(\Delta_{D}\right)^{m}$. By induction this holds for all $f \in I^{(m)}$. The induction step assumes $n \geq 3$. The base cases $n=1,2$ are trivial.

Finally, we discuss property (4). Take the Rees algebra $R=\mathbb{C}[\boldsymbol{x}, \boldsymbol{y}]\left[t\left(\Delta_{D}\right)\right]$, and put $X=\operatorname{Proj} R$, that is, the blowup of $E$ at the ideal $\left(\Delta_{D}\right)$. Here, as above, $\left(\Delta_{D}\right)$ is shorthand for the ideal generated by all the alternating polynomials $\Delta_{D}$. 
In view of Theorem 1.1, we can also identify $X$ with the blowup of $E$ along $V$, but it is preferable for geometric reasons not to take this as the definition.

The symmetric group $S_{n}$ acts equivariantly on both $X$ and $E$, giving a diagram

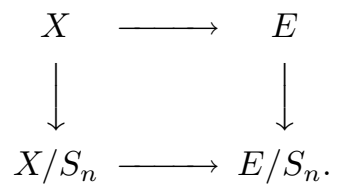

Now it develops that $X / S_{n}$ is nothing else but the Hilbert scheme $\operatorname{Hilb}^{n}\left(\mathbb{C}^{2}\right)$ parametrizing 0 -dimensional subschemes of length $n$ in $\mathbb{C}^{2}$, or equivalently, ideals $\mathscr{J} \subseteq \mathbb{C}[x, y]$ such that $\operatorname{dim}_{\mathbb{C}} \mathbb{C}[x, y] / \mathscr{J}=n$. This is in fact not difficult to show, using explicit local coordinates on $\operatorname{Hilb}^{n}\left(\mathbb{C}^{2}\right)$ and the definition of $X$.

By a classical theorem of Fogarty [1968], $\operatorname{Hilb}^{n}\left(\mathbb{C}^{2}\right)$ is non-singular and irreducible - see the Appendix for another proof using explicit local coordinates. It is also known that the locus in $\operatorname{Hilb}^{n}\left(\mathbb{C}^{2}\right)$ where the $y$-coordinates vanish, that is, the locus describing subschemes of $\mathbb{C}^{2}$ supported on the $x$-axis, has codimension $n$. From this it follows easily that $\operatorname{dim} R /(\boldsymbol{y})=n+1$.

We come now to the most important theorem from the geometric point of view.

Theorem 1.10. The blowup scheme $X$ is arithmetically Gorenstein, that is, $R$ is a Gorenstein ring.

Let us pause to understand how this result is related to Theorem 1.8. The dimension count above shows that $(\boldsymbol{y})$ is a complete intersection ideal in $R$. Hence, if we assume Theorem 1.10 holds, then $R$ is a free $\mathbb{C}[\boldsymbol{y}]$-module, which is merely a restatement of Theorem 1.8. So Theorem 1.8 is a simple corollary to Theorem 1.10.

Unfortunately for this logic, the only proof of Theorem 1.10 known at present uses Theorem 1.8. Specifically, although the main argument of the proof given in [Haiman 2001] is an induction based on elementary geometry of the Hilbert schemes, there is a key technical step that depends on Theorem 1.8. So for now we cannot elegantly deduce Theorem 1.8 from Theorem 1.10, as above, but must prove Theorem 1.8 directly.

Problem 1.11. Find an "intrinsic" proof of Theorem 1.10 that does not rely on Theorem 1.8.

In this connection we may note that there are classical theorems in commutative algebra for showing that Rees algebras are Cohen-Macaulay or Gorenstein. In particular, as W. Vasconcelos pointed out to me, since our ideal has codimension 2 it is enough to show that the Rees algebra $R$ is Cohen-Macaulay, and it is then automatically Gorenstein (this consequence also follows from the geometry). Unfortunately, as far as I am aware, the theorems one might use to show that $R$ is Cohen-Macaulay tend to require hypotheses on the blowup ideal, such as strong 
Cohen-Macaulayness, or small analytic spread, that fail drastically for our ideal $I$. It is natural to inquire whether advances in singularity theory might even make it possible to show that our Rees algebra $R$ has singularities better than Cohen-Macaulay. Could one hope to prove, for instance, that $R$ is of $F$-rational type?

I'll conclude with some remarks concerning the existing proof and possible generalizations of Theorem 1.8, which for the moment remains the linchpin among the results. To prove Theorem 1.8, we first show that $\left(\Delta_{D}\right)^{m}$ is a direct summand as a graded $\mathbb{C}[\boldsymbol{x}, \boldsymbol{y}]$-module of the coordinate ring $\mathbb{C}[W]$ of an auxiliary subspace arrangement $W \subseteq E \times \mathbb{C}^{2 m n}$, called a "polygraph." Then we show that $\mathbb{C}[W]$ is a free $\mathbb{C}[\boldsymbol{y}]$-module by explicitly constructing a basis. This requires a horribly complicated and not very illuminating induction. The basis construction is secretly modeled on a combinatorial interpretation of a formula from geometry for the Hilbert series of $\mathbb{C}[W]$. In the end, however, both the formula and the combinatorics are suppressed from the proof, as they must be, since one can only prove such formulas by assuming the theorem a priori.

I think that some of the complexity of the existing proof may eventually be removed. I also think that most of the phenomena concerning the ideal $I$ should persist if we take points in $\mathbb{C}^{d}$ for general $d$, instead of $\mathbb{C}^{2}$. If so, we will need proofs that do not refer to the Hilbert scheme, secretly or otherwise. Here are some specific problems motivated by my thoughts along these lines.

PROBLEM 1.12. Is it possible to dispense with the polygraph and construct a free $\mathbb{C}[\boldsymbol{y}]$-module basis of $\left(\Delta_{D}\right)^{m}$ directly? It would already be interesting to accomplish this for $d=2$. In this case, the geometry does provide a formula for the Hilbert series, but an obstacle to using it is that we don't have a combinatorial interpretation, and therefore no clue how to index the basis elements.

Problem 1.13. Our subspace arrangement $V$ can be written as $\mathbb{C}^{2} \otimes V^{\prime}$, where $V^{\prime}$ is the hyperplane arrangement $V^{\prime}=\bigcup_{i<j} V\left(x_{i}-x_{j}\right)$ in $\mathbb{C}^{n}$. Here, for any subspace arrangement $A=\bigcup_{k} A_{k} \subseteq \mathbb{C}^{n}$, we denote by $\mathbb{C}^{d} \otimes A$ the arrangement of subspaces $\mathbb{C}^{d} \otimes A_{k} \subseteq \mathbb{C}^{d} \otimes \mathbb{C}^{n}=\mathbb{C}^{d n}$.

(a) Is it true more generally that for all $d$, the ideal of $\mathbb{C}^{d} \otimes V^{\prime}$ is a free $\mathbb{C}[\boldsymbol{x}]$ module, where $\boldsymbol{x}$ is one of the $d$ sets of $n$ coordinates on $\mathbb{C}^{d n}$ ?

(b) The hyperplane arrangement $V^{\prime}$ is the Coxeter arrangement of type $A_{n-1}$. What if we consider instead the Coxeter arrangements of other types?

(c) Are there general criteria for a hyperplane arrangement $A \subseteq \mathbb{C}^{n}$ to have the property that the ideal $I_{d}$ of $\mathbb{C}^{d} \otimes A$ is a free module over the coordinate ring of $\mathbb{C}^{n}$, for all $d$ ?

(d) Exercise: show that a hyperplane arrangement with the property in (c) must be free in the sense used in the theory of hyperplane arrangements [Orlik and Terao 1992]. Freeness as a hyperplane arrangement is not sufficient for (c), however. 


\section{Lecture 2: A Ring of Invariants}

As in Lecture 1, let $E=\mathbb{C}^{2 n}$ be the space of $n$-tuples $\left(P_{1}, \ldots, P_{n}\right)$ of points in the plane. The action of the symmetric group $S_{n}$ on $E$ has already made an appearance in our study of the ideal of the locus where points coincide. In this lecture we will discuss some other features of this action. We will begin with a review of some general theory of invariants and coinvariants of linear representations of finite groups, then turn to particulars of the representation of $S_{n}$ on $E$.

For the moment, we consider an arbitrary finite group $G$, acting linearly on a finite-dimensional vector space $V=k^{n}$. Our only assumption will be that char $k$ does not divide $|G|$. Then all finite-dimensional representations of $G$ are completely reducible, that is, they are direct sums of irreducible representations. In particular, each homogeneous component of the ring $k[V]$ of polynomial functions on $V$ is completely reducible. Of special interest is the subring of invariants $k[V]^{G}$. It follows from complete reducibility that $k[V]^{G}$ is a direct summand of $k[V]$ as a $G$-module, and also as a $k[V]^{G}$-module. The projection of $k[V]$ on its summand $k[V]^{G}$ is given explicitly by the Reynolds operator

$$
\boldsymbol{R} f=\frac{1}{|G|} \sum_{g \in G} g \cdot f
$$

which will be important in what follows.

A second ring associated with the action of $G$ on $V$ is the ring of coinvariants , defined as

$$
R_{G}=k[V] / I_{G},
$$

where $I_{G}=k[V] \cdot\left(k[V]_{+}^{G}\right)$ is the ideal generated by all homogeneous invariants of positive degree. Geometrically, these rings have the following interpretation (at least when $k$ is algebraically closed). The space of $G$-orbits $V / G$ has a natural structure of algebraic variety, with regular functions given by the $G$-invariant functions on $V$. Thus its coordinate ring is the ring of invariants:

$$
k[V]^{G}=k[V / G] .
$$

The homogeneous maximal ideal $k[V]_{+}^{G}$ in $k[V]^{G}$ is the ideal of the origin $0 \in V / G$ (the $G$-orbit consisting only of the origin in $V$ ). Then the scheme-theoretic fiber $\pi^{-1}(0)$ of the natural projection

$$
\pi: V \rightarrow V / G
$$

has coordinate ring equal to the ring of coinvariants,

$$
R_{G}=k\left[\pi^{-1}(0)\right] \text {. }
$$

The two constructions are related by a famous lemma of Hilbert.

Lemma 2.1 (Hilbert). Homogeneous invariants $f_{1}, \ldots, f_{r}$ of positive degree generate $k[V]^{G}$ as a $k$-algebra if and only if they generate $I_{G}$ as an ideal. 
Proof. If $k[V]^{G}=k\left[f_{1}, \ldots, f_{r}\right]$, then every homogeneous invariant of positive degree is a polynomial without constant term in the $f_{i}$ 's. This shows that $I_{G} \subseteq$ $\left(f_{1}, \ldots, f_{r}\right)$, and the reverse inclusion is trivial.

For the converse, suppose to the contrary that $I_{G}=\left(f_{1}, \ldots, f_{r}\right)$ but $k[V]^{G} \neq$ $k\left[f_{1}, \ldots, f_{r}\right]$. Let $h$ be a homogeneous invariant of minimal degree, say $d$, not contained in $k\left[f_{1}, \ldots, f_{r}\right]$. Certainly $d>0$, so $h \in I_{G}$, and we can write

$$
h=\sum_{i} a_{i} f_{i}
$$

where we can assume without loss of generality that $a_{i}$ is homogeneous of degree $d-\operatorname{deg} f_{i}$. Applying the Reynolds operator to both sides gives

$$
h=\sum_{i}\left(\boldsymbol{R} a_{i}\right) f_{i} .
$$

But each $\boldsymbol{R} a_{i}$ is a homogeneous invariant of degree $<d$, hence belongs to $k\left[f_{1}, \ldots, f_{r}\right]$. This contradicts the assumption $h \notin k\left[f_{1}, \ldots, f_{r}\right]$.

It is natural to ask for a bound on the degrees of a minimal set of homogeneous generators for $k[V]^{G}$, or equivalently for $I_{G}$. To give precise bounds for particular $G$ and $V$ is in general a difficult problem. One has the following global bound, which was proved by Noether in characteristic 0 .

THEOREM 2.2. The ring of invariants $k[V]^{G}$ is generated by homogeneous elements of degree at most $|G|$.

Let us pause to discuss a more modern proof of this theorem, based on a beautiful lemma of Harm Derksen. To state the lemma we need some additional notation. Let $x_{1}, \ldots, x_{n}$ be a basis of coordinates on $V$, so $k[V]=k[\boldsymbol{x}]$. We introduce a second copy of $V$, with coordinates $y_{1}, \ldots, y_{n}$. Then the coordinate ring $k[V \times V]$ is identified with the polynomial ring $k[\boldsymbol{x}, \boldsymbol{y}]$. For each $g \in G$, let

$$
J_{g}=\left(x_{i}-g y_{i}: 1 \leq i \leq n\right) \subseteq k[\boldsymbol{x}, \boldsymbol{y}]
$$

be the ideal of the subspace $W_{g}=\{(v, g v): v \in V\} \subseteq V \times V$.

Lemma 2.3 [Derksen 1999]. Let $J=\bigcap_{g \in G} J_{g}$, with $J_{g}$ as above. Then $k[\boldsymbol{x}] \cap$ $(J+(\boldsymbol{y}))=I_{G}$.

Proof. If $f(\boldsymbol{x})$ is a homogeneous invariant of positive degree, then $f(\boldsymbol{y}) \in(\boldsymbol{y})$, and $f(\boldsymbol{x})-f(\boldsymbol{y}) \in J$, since $f(\boldsymbol{x})-f(\boldsymbol{y})$ vanishes on setting $\boldsymbol{y}=g \boldsymbol{x}$ for any $g \in G$. This shows $I_{G} \subseteq k[\boldsymbol{x}] \cap(J+(\boldsymbol{y}))$.

For the reverse inclusion, suppose $f(\boldsymbol{x}) \in J+(\boldsymbol{y})$, so

$$
f(\boldsymbol{x})=\sum_{i} a_{i}(\boldsymbol{x}) b_{i}(\boldsymbol{y})+p(\boldsymbol{x}, \boldsymbol{y}),
$$

where $p(\boldsymbol{x}, \boldsymbol{y}) \in J$ and we can assume $b_{i}(\boldsymbol{y})$ homogeneous of positive degree. Let $\boldsymbol{R}_{\boldsymbol{y}}$ be the Reynolds operator for the action of $G$ on the $\boldsymbol{y}$ variables only. The 
ideal $J$ is invariant for this action, so $\boldsymbol{R}_{\boldsymbol{y}} J \subseteq J$. Hence, applying $\boldsymbol{R}_{\boldsymbol{y}}$ to both sides in $(2-2)$ yields

$$
f(\boldsymbol{x})=\sum_{i} a_{i}(\boldsymbol{x}) \boldsymbol{R}_{\boldsymbol{y}} b_{i}(\boldsymbol{y})+q(\boldsymbol{x}, \boldsymbol{y})
$$

with $q(\boldsymbol{x}, \boldsymbol{y}) \in J$. In particular, $q(\boldsymbol{x}, \boldsymbol{x})=0$. Substituting $\boldsymbol{y} \mapsto \boldsymbol{x}$ on both sides now exhibits $f$ as an element of $I_{G}$.

We remark that $J$ is the ideal of the subspace arrangement $W=\bigcup_{g} W_{g}$, which we will call Derksen's arrangement. It is the arrangement in $V \times V$ whose projection on the first factor $V$ has finite fiber over each point $v$, identified set-theoretically with the orbit $G v$ (by projecting on the second factor). Derksen's Lemma says that the scheme-theoretic 0-fiber of the projection $W \rightarrow V$ is isomorphic to the scheme-theoretic 0-fiber of $\pi: V \rightarrow V / G$, that is, to $\operatorname{Spec} R_{G}$.

Derksen's lemma has the following easy analog for the product ideal.

Lemma 2.4. Let $d=|G|$ and let $J^{\prime}=\prod_{g} J_{g}$, with $J_{g}$ as in (2-1). Then $k[\boldsymbol{x}] \cap\left(J^{\prime}+(\boldsymbol{y})\right)=(\boldsymbol{x})^{d}$.

Proof. Note that $k[\boldsymbol{x}] \cap\left(J^{\prime}+(\boldsymbol{y})\right)$ is the set of polynomials $\{f(\boldsymbol{x}, 0): f(\boldsymbol{x}, \boldsymbol{y}) \in$ $\left.J^{\prime}\right\}$ (this holds with any ideal in the role of $J^{\prime}$ ). Since $J^{\prime}$ is generated by products of $d$ linear forms, this shows $k[\boldsymbol{x}] \cap\left(J^{\prime}+(\boldsymbol{y})\right) \subseteq(\boldsymbol{x})^{d}$. For the reverse inclusion, fix any monomial $\boldsymbol{x}^{\alpha}$ of degree $d$, and write it as a product of individual variables

$$
\boldsymbol{x}^{\alpha}=x_{i_{1}} x_{i_{2}} \ldots x_{i_{d}} .
$$

Let $g_{1}, \ldots, g_{d}$ be an enumeration of all the elements of $G$, and consider the polynomial

$$
f(\boldsymbol{x}, \boldsymbol{y})=\prod_{j}\left(x_{i_{j}}-g_{j} y_{i_{j}}\right) .
$$

The $j$-th factor belongs to $J_{g_{j}}$, so $f(\boldsymbol{x}, \boldsymbol{y}) \in J^{\prime}$, and clearly $f(\boldsymbol{x}, 0)=\boldsymbol{x}^{\alpha}$.

Now $J^{\prime} \subseteq J$, so Lemmas 2.3 and 2.4 imply $(\boldsymbol{x})^{d} \subseteq I_{G}$. Hence $I_{G}$ is generated by its homogeneous elements of degree at most $d$, proving Theorem 2.2. In fact, we have proved something stronger.

Corollary 2.5. The ring of coinvariants $R_{G}$ is zero in degrees $\geq|G|$.

The degree bound in Theorem 2.2 is tight only when $G$ is a cyclic group. For arbitrary $G$ and $V$, rather little is known about how to describe $k[V]^{G}$ and $R_{G}$ more fully. Of the two, the ring of invariants is better understood. In particular, we have the Eagon-Hochster theorem:

Theorem 2.6 [Hochster and Eagon 1971]. The ring of invariants $k[V]^{G}$ is Cohen-Macaulay.

My hope in this lecture is to persuade you that $k[V]^{G}$ and $R_{G}$ can have surprisingly rich structure for naturally occurring group representations, and that the 
problem of describing them is deserving of further study. We now turn to the particular case $G=S_{n}$, and fix $k=\mathbb{C}$. As we did in Lecture 1 , let's warm up in the easier situation of $n$ points on a line. This means we consider the representation of $S_{n}$ on $V=\mathbb{C}^{n}$, permuting the coordinates $x_{1}, \ldots, x_{n}$. We make several observations.

(I) The ring of invariants $\mathbb{C}[\boldsymbol{x}]^{S_{n}}$ is the polynomial ring $\mathbb{C}\left[e_{1}, \ldots, e_{n}\right]$ freely generated by the elementary symmetric functions $e_{j}=e_{j}(\boldsymbol{x})$. This is the fundamental theorem of symmetric functions. Its Hilbert series is

$$
\frac{1}{(1-q)\left(1-q^{2}\right) \cdots\left(1-q^{n}\right)}
$$

which can also be written as

$$
h_{n}\left(1, q, q^{2}, \ldots\right)
$$

where $h_{n}\left(z_{1}, z_{2}, \ldots\right)$ denotes the complete homogeneous symmetric function of degree $n$ in infinitely many variables.

(II) By Lemma 2.1, $I_{S_{n}}(\boldsymbol{x})=\left(e_{1}, \ldots, e_{n}\right)$. In particular it is a complete intersection ideal. Hence $R_{S_{n}}(\boldsymbol{x})$ is an Artinian local complete intersection ring. It can be described quite precisely. For example, since $\operatorname{deg} e_{j}=j$, the Hilbert series of $R_{S_{n}}(\boldsymbol{x})$ is given by the $q$-analog of $n$ !, namely,

$$
[n]_{q} !=\frac{(1-q)\left(1-q^{2}\right) \cdots\left(1-q^{n}\right)}{(1-q)^{n}}=[n]_{q}[n-1]_{q} \ldots[1]_{q},
$$

where $[k]_{q}=1+q+\cdots+q^{k-1}$. Hence

$$
\operatorname{dim}_{\mathbb{C}} R_{S_{n}}(\boldsymbol{x})=n !
$$

(III) Since $\mathbb{C}[\boldsymbol{x}]$ is a graded Cohen-Macaulay ring, and $e_{1}, \ldots, e_{n}$ is a homogeneous system of parameters, it follows that $\mathbb{C}[\boldsymbol{x}]$ is a free $\mathbb{C}[\boldsymbol{x}]^{S_{n}}$-module, with basis given by any $n$ ! homogeneous elements forming a vector space basis of $R_{S_{n}}(\boldsymbol{x})$. It is easy using standard techniques to determine the character of the polynomial ring $\mathbb{C}[\boldsymbol{x}]$ as a graded $S_{n}$ representation, and from this to determine the corresponding graded character of $R_{S_{n}}(\boldsymbol{x})$. The answer can be expressed as follows. The irreducible representations $V_{\lambda}$ of $S_{n}$ are indexed by partitions $\lambda$ of the integer $n$. For each $\lambda$, define

$$
f_{\lambda}(q)=(1-q)\left(1-q^{2}\right) \cdots\left(1-q^{n}\right) s_{\lambda}\left(1, q, q^{2}, \ldots\right)
$$

where $s_{\lambda}\left(z_{1}, z_{2}, \ldots\right)$ is the Schur symmetric function indexed by $\lambda$ in infinitely many variables. Then $f_{\lambda}(q)$ is a polynomial with positive integer coefficients, and $f_{\lambda}(1)$ is the number of standard Young tableau of shape $\lambda$, which is also equal to $\operatorname{dim} V_{\lambda}$. Let $m\left(V_{\lambda}, R_{S_{n}}(\boldsymbol{x})_{d}\right)$ denote the multiplicity of $V_{\lambda}$ in a decomposition 
of the degree $d$ homogeneous component $R_{S_{n}}(\boldsymbol{x})_{d}$ as a direct sum of irreducible representations of $S_{n}$. Then these multiplicities are given by

$$
\sum_{d} m\left(V_{\lambda}, R_{S_{n}}(\boldsymbol{x})_{d}\right) q^{d}=f_{\lambda}(q)
$$

This is a very precise answer, as $f_{\lambda}(q)$ has an explicit combinatorial description, and it is possible to produce a correspondingly explicit decomposition of $R_{S_{n}}(\boldsymbol{x})$ into irreducibles with generators indexed by suitable combinatorial data. It would take us too far afield to go into this here, but see [Allen 1993], for example, for more details. We only note that ignoring the grading gives

$$
m\left(V_{\lambda}, R_{S_{n}}(\boldsymbol{x})\right)=f_{\lambda}(1)=\operatorname{dim} V_{\lambda},
$$

so $R_{S_{n}}(\boldsymbol{x})$ is a graded version of the regular representation of $S_{n}$ (the representation of $S_{n}$ by left multiplication on its group algebra $\left.\mathbb{C} S_{n}\right)$.

(IV) Derksen's arrangement $W$ is a complete intersection in $\mathbb{C}^{n} \times \mathbb{C}^{n}$, defined by the ideal $\left(e_{i}(\boldsymbol{x})-e_{i}(\boldsymbol{y}): 1 \leq i \leq n\right)$. In particular, its coordinate ring $\mathbb{C}[W]$ is Cohen-Macaulay, and since $(\boldsymbol{y})$ is obviously a system of parameters, $\mathbb{C}[W]$ is a free $\mathbb{C}[\boldsymbol{y}]$-module.

These special properties of the invariants and coinvariants of $S_{n}$ on $\mathbb{C}^{n}$ are consequences of the fact that $S_{n}$ acts on $\mathbb{C}^{n}$ as a group generated by complex reflections: linear transformations that fix a hyperplane pointwise. In the case of $S_{n}$, the reflections are the transpositions $(i, j)$, which fix every vector on the hyperplane $x_{i}=x_{j}$. By general results of Steinberg, Chevalley, Shepard and Todd, every complex reflection group $G$ has $k[V]^{G}$ a polynomial ring, $I_{G}$ a complete intersection ideal, and $R_{G}$ isomorphic to a graded version of the regular representation of $G$. Moreover, each of these properties holds only for complex reflection groups, and there is a complete classification of such groups [Chevalley 1955; Shephard and Todd 1954; Steinberg 1960; 1964].

Finally we come to the situation that we set out to study in the first place, namely, the action of $S_{n}$ on $E=\mathbb{C}^{2 n}$. Note that this is not an action generated by complex reflections. In fact, every element of $S_{n}$ acts on $E$ with determinant 1 , while a nontrivial complex reflection has determinant $\neq 1$. The determinant 1 property does have a useful consequence, however, owing to the following refinement of the Eagon-Hochster theorem.

THEOREM 2.7 [Watanabe 1974]. The canonical module of $k[V]^{G}$ is the module of covariants $k[V]^{\varepsilon}$, where $\varepsilon$ denotes the determinant character $\varepsilon(g)=\operatorname{det}_{V}(g)$. In particular if $G$ acts on $V$ by endomorphisms with determinant 1 , then $k[V]^{G}$ is Gorenstein.

There is an old theorem of Weyl giving a (minimal) generating set for the ring of invariants $\mathbb{C}[E]^{S_{n}}$. 
Theorem 2.8 [Weyl 1939]. The ring of invariants $\mathbb{C}[\boldsymbol{x}, \boldsymbol{y}]^{S_{n}}$ is generated by the polarized power sums

$$
p_{r, s}=\sum_{i=1}^{n} x_{i}^{r} y_{i}^{s}, \quad 1 \leq r+s \leq n .
$$

The analogous theorem holds in $d$ sets of variables. Note that the actual degree bound on the generators in this case, namely $n$, is very much smaller than the order of the group!

It turns out to be almost as easy to determine the Hilbert series of $\mathbb{C}[E]^{S_{n}}=$ $\mathbb{C}[\boldsymbol{x}, \boldsymbol{y}]^{S_{n}}$ as it is for $\mathbb{C}[\boldsymbol{x}]^{S_{n}}$. In fact, we can compute its Hilbert series as a doubly graded ring, by degree in the $\boldsymbol{x}$ and $\boldsymbol{y}$ variables separately. It is given by the following analog of $(2-3)$.

$$
\sum_{r, s} \operatorname{dim}\left(\mathbb{C}[\boldsymbol{x}, \boldsymbol{y}]^{S_{n}}\right)_{r, s} q^{r} t^{s}=h_{n}\left(1, q, q^{2}, \ldots, t, q t, q^{2} t, \ldots, t^{2}, q t^{2}, q^{2} t^{2}, \ldots\right) .
$$

There is a also similar formula for the Hilbert series of the ring of invariants $\mathbb{C}[\boldsymbol{x}, \boldsymbol{y}, \ldots, \boldsymbol{z}]^{S_{n}}$ in $d$ sets of variables, as an $\mathbb{N}^{d}$-graded ring. So we have good analogs of observation (I) for the invariants of $n$ points in the plane or more generally in $\mathbb{C}^{d}$.

The interesting surprises appear when we turn to analogs of observations (II) and (III), on the ring of coinvariants. We now drop the modifier $\boldsymbol{x}$ from the notation and write simply $R_{S_{n}}$ for the ring of coinvariants $\mathbb{C}[E] / I_{S_{n}}$.

Around 1991, Garsia and I were led to investigate $R_{S_{n}}$ because of its connection with a problem on Macdonald polynomials. For small values of $n$, we used a computer to determine its dimension and $S_{n}$ character in each (double) degree. Immediately we noticed some amazing coincidences between our data and well-known combinatorial numbers. We publicized our early findings informally, leading various other people, especially Ira Gessel and Richard Stanley, to discover still more such coincidences. Eventually I published a compilation of these discoveries, all of which were then just conjectures, in [Haiman 1994].

Later, Procesi pointed out to us the fact that the Hilbert scheme Hilb ${ }^{n}\left(\mathbb{C}^{2}\right)$ provides a nice resolution of singularities of $E / S_{n}$, as discussed in Lecture 1 , and observed how this should be useful in attacking the conjectures. Assuming the validity of some geometric hypotheses that would make Procesi's method work, I was soon able to find a formula for the doubly graded character of $R_{S_{n}}$ in terms of Macdonald polynomials. Garsia and I then proved that the earlier combinatorial conjectures would all follow from the master formula. Recently I succeeded in proving the needed geometric hypotheses, which by this time were the only missing pieces remaining [Haiman 2001; 2002].

There is not room here to discuss in full the geometry of the Hilbert scheme and the combinatorial theory of Macdonald polynomials. I will only summarize some of the facts about $R_{S_{n}}$ that have been established using these methods. 
THEOREM 2.9. The coinvariant ring $R_{S_{n}}$ for $S_{n}$ acting on $\mathbb{C}^{2 n}$ has length

$$
\operatorname{dim}_{\mathbb{C}} R_{S_{n}}=(n+1)^{n-1} .
$$

Ignoring the grading, the representation of $S_{n}$ on $R_{S_{n}}$ is isomorphic to the sign representation tensored by the obvious permutation representation of $S_{n}$ on the finite Abelian group $Q /(n+1) Q$, where $Q=\mathbb{Z}^{n} / \mathbb{Z} \cdot(1,1, \ldots, 1)$. Retaining the grading by $\boldsymbol{x}$ degree only, one has the Hilbert series

$$
\sum_{d} \operatorname{dim}\left(R_{S_{n}}\right)_{(d,-)} q^{d}=F_{n}(q)
$$

where $F_{n}(q)$ is the generating function enumerating rooted forests on the vertex set $\{1, \ldots, n\}$ by number of inversions, or equivalently, enumerating parking functions on $n$ cars by weight (see [Haiman 1994] for definitions and details).

Here we should mention the connection between $R_{S_{n}}$ and the ideal $I$ studied in the previous lecture, given by the following proposition, which is easy to prove.

Proposition 2.10. Homogeneous $S_{n}$-alternating polynomials $f_{1}, \ldots, f_{r} \in \mathbb{C}[E]$ minimally generate the ideal I in Theorem 1.1 if and only if their images modulo $I_{S_{n}}$ form a basis of the space of $S_{n}$-alternating elements of $R_{S_{n}}$.

In particular, Theorem 1.2 is really a statement about the character of $R_{S_{n}}$. Like Theorem 2.9, it follows from the master formula for the character of $R_{S_{n}}$ given by the geometry of the Hilbert scheme.

I think it should be possible to obtain at least some of the above results on $R_{S_{n}}$, and maybe some new ones, or analogous ones for other groups, without invoking Hilbert scheme and Macdonald polynomial machinery. In particular, it seems to me that there is room for purely algebraic approaches. One encouraging sign is recent work by Iain Gordon [2003], where he obtains an extension of the $(n+1)^{n-1}$ theorem, in a slightly weakened form, to any Weyl group. This is especially notable in that for the Weyl groups of type $G_{2}, F_{4}$, and $D_{n}$, it is known that there is no suitable geometric analog of the Hilbert scheme.

To close, let me suggest some open problems that might repay further study.

Problem 2.11. Can one determine the dimension and Hilbert series of $R_{S_{n}}$ inductively by fitting it into an exact complex with other terms built out of the coinvariant rings $R_{S_{k}}$ for $k<n$ ? A specific conjecture along these lines in [Haiman 1994] remains open.

Problem 2.12. Describe the minimal free resolution of $\mathbb{C}[\boldsymbol{x}, \boldsymbol{y}]^{S_{n}}$ with respect to the minimal generators given by Theorem 2.8. One could also consider this problem in $d$ sets of variables, although $d=2$ may be nicer, since the ring of invariants is Gorenstein. I don't think a good description is known even for the first syzygies. 
Problem 2.13. Let $W$ be the Derksen arrangement for $S_{n}$ acting on $E$, say with coordinates $\boldsymbol{x}, \boldsymbol{y}, \boldsymbol{x}^{\prime}, \boldsymbol{y}^{\prime}$ on $E \times E$. Is $\mathbb{C}[W]$ a free $\mathbb{C}[\boldsymbol{y}]$-module? What about the same problem for fiber powers $W \times_{E} W \times_{E} \cdots \times{ }_{E} W$ ? An affirmative answer would be equivalent to sheaf cohomology vanishing properties for certain vector bundles on the Hilbert scheme. Are there similar results in $d$ sets of variables, with $E$ replaced by $\mathbb{C}^{d n}$ ? Are there similar results for other Weyl groups $G$, with $E$ the direct sum of two (or more) copies of the defining representation?

\section{Lecture 3: A Remarkable Gröbner Basis}

This lecture will be an overview of some results by Jeremy Martin. I'll give less detailed notes here than for the previous two lectures, referring you to [Martin 2003a; 2003b] for the full story. Martin's results concern the situation where we introduce not only the points $P_{1}, \ldots, P_{n} \in \mathbb{C}^{2}$ but also lines $L_{i j}$ connecting them in pairs. That is, $L_{i j}$ is a line passing through $P_{i}$ and $P_{j}$. When $P_{i}$ and $P_{j}$ are distinct, of course, $L_{i j}$ is determined. When they coincide, the line $L_{i j}$ can pass through them with any slope, introducing an extra degree of freedom.

The locus of all configurations of points and lines as above is the picture space $\mathscr{X}\left(K_{n}\right)$. One thinks of these configurations as plane "pictures" of the complete graph $K_{n}$ on $n$ vertices, with edges represented by lines. To specify a picture, we need to give the coordinates $x_{1}, y_{1}, \ldots, x_{n}, y_{n}$ of the $n$ points, together with the slopes $m_{i j}$ of the $\left(\begin{array}{l}n \\ 2\end{array}\right)$ lines. In principle, the slopes $m_{i j}$ lie on a projective line $\mathbb{P}^{1}$. However, we will be interested only in local questions, so we will consider the affine open set in $\mathscr{X}\left(K_{n}\right)$ where $m_{i j} \neq \infty$. It is the locus cut out (settheoretically, at least) by the equations

$$
y_{j}-y_{i}=m_{i j}\left(x_{j}-x_{i}\right) \text { for all } i, j .
$$

Now $\mathscr{X}\left(K_{n}\right)$ is in general not irreducible. For example, $\mathscr{X}\left(K_{4}\right)$ has two irreducible components, each of dimension 8: the generic component - the closure of the locus where all the points are distinct, and the lines are determined and another component where all four points coincide, and the six lines have arbitrary slopes. Martin has given a complete combinatorial description of the component structure of $\mathscr{X}(G)$ for any graph $G$, which we won't discuss in this lecture. Instead we will concentrate on his results describing the generic component $\mathscr{V}\left(K_{n}\right)$ of $\mathscr{X}\left(K_{n}\right)$, which we call the graph variety. Note that $\mathscr{V}\left(K_{n}\right)$ is, essentially by definition, the simultaneous blowup of $\mathbb{C}^{2 n}$ along the coincidence subspaces $V_{i j}=V\left(x_{i}-x_{j}, y_{i}-y_{j}\right)$ discussed in Lecture 1 . This is, however, quite a different thing from the blowup along the union of these subspaces, which is the variety $X$ from Lecture 1 .

Proposition 2.1. The graph variety $\mathscr{V}\left(K_{n}\right)$ is cut out set-theoretically in $\mathscr{X}\left(K_{n}\right)$ by the equations in the variables $m_{i j}$ giving the algebraic relations among the slopes that hold when the points $P_{i}$ are in general position (no two coincide). 
In view of this proposition, the key issue is to understand the ideal of relations among the slope variables $m_{i j}$. Although the problem of describing all relations among the slopes of the $\left(\begin{array}{l}n \\ 2\end{array}\right)$ lines connecting $n$ points in general position in the plane is very classical in nature, there seems to have been almost no earlier work on it. In more geometric terms, the projection of the graph variety $\mathscr{V}\left(K_{n}\right)$ on the slope coordinates is a variety $\mathscr{S}\left(K_{n}\right)$, called the slope variety, whose ideal $I\left(\mathscr{S}_{n}\right)$ is the ideal of all algebraic dependencies among the rational functions $\left(y_{j}-y_{i}\right) /\left(x_{j}-x_{i}\right)$. We want to describe this ideal.

The first result tells us which subsets of the variables $m_{i j}$ are minimally algebraically dependent - that is, are circuits of the algebraic dependence matroid of the quantities $\left(y_{j}-y_{i}\right) /\left(x_{j}-x_{i}\right)$.

TheOREM 2.2. The variables $m_{i j}$ corresponding to a set of edges $E \subseteq E\left(K_{n}\right)$ are minimally algebraically dependent if and only if

(1) $|E|=2|V(E)|-2$, and

(2) $|F| \leq 2|V(F)|-3$ for all nonempty $F \varsubsetneqq E$,

where $V(E)$ denotes the set of all endpoints of the edges in $E$.

This result is particularly interesting because there is another well-known algebraic dependence matroid whose characterization (due to Laman) is exactly the same: that is the rigidity matroid of algebraic dependencies among the squaredlengths $\left(x_{i}-x_{j}\right)^{2}+\left(y_{i}-y_{j}\right)^{2}$ of the line segments connecting the points (for points with real coordinates).

The next result, which is a key one, is an explicit description of the polynomial giving the algebraic dependence among the slopes in a rigidity-circuit. First one shows that every rigidity circuit is the edge-disjoint union of two spanning trees on a common set of vertices. Conversely, every minimal such union is a rigidity circuit.

Now consider any two disjoint spanning trees $S$ and $T$ on the same vertex set, and fix an arbitrary orientation of the edges of each tree. For each edge $f \in S$, there are unique coefficients $c_{e f} \in\{0, \pm 1\}$ such that

$$
f-\sum_{e \in T} c_{e f} e
$$

is a directed cycle. Let us abbreviate $x_{e}=x_{j}-x_{i}, y_{e}=y_{j}-y_{i}$ for a directed edge $e=(i, j)$. Then for a cycle as in $(2-1)$, we have

$$
y_{f}=\sum_{e \in T} c_{e f} y_{e}, \quad x_{f}=\sum_{e \in T} c_{e f} x_{e} .
$$

Now since $y_{f}=m_{f} x_{f}$ and $y_{e}=m_{e} x_{e}$, we have an identity between two expressions for $y_{f}$

$$
\sum_{e \in T} c_{e f} m_{e} x_{e}=m_{f} \sum_{e \in T} c_{e f} x_{e}
$$


or

$$
\sum_{e \in T} c_{e f}\left(m_{e}-m_{f}\right) x_{e}=0
$$

This of course is not yet an equation among the variables $m_{i j}$. However, if $S$ and $T$ are trees on $d+1$ vertices, then we have $d$ such equations, one for each $f$, which we can regard as linear equations in the $d$ "unknowns" $x_{e}$. When the points are in general position, they obviously have a nonzero solution, since the $x_{e}$ 's do not vanish. Hence the $d \times d$ matrix

$$
M_{S T}=\left[c_{e f}\left(m_{e}-m_{f}\right)\right]_{f \in S, e \in T}
$$

must be singular. Its determinant

$$
D_{S \cup T}(m)
$$

is a polynomial of degree $d$ in the slope variables $m_{e}$ for $e$ in our rigidity circuit $S \cup T$, and this polynomial belongs to $I\left(\mathscr{S}_{n}\right)$.

TheOREM 2.3. The determinants $D_{S \cup T}$ enjoy the following properties:

(1) Up to sign, $D_{S \cup T}$ depends only on the union $S \cup T$, and not on the decomposition into trees $S, T$.

(2) Every term of $D_{S \cup T}$ is a square-free monomial $\pm \prod_{e \in S^{\prime}} m_{e}$, where $S^{\prime}$ is a spanning tree in $S \cup T$ whose complement is also a spanning tree.

(3) $D_{S \cup T}$ is irreducible if and only if $S \cup T$ is a rigidity circuit, and in that case it generates the principal ideal of algebraic dependencies among the slope variables $m_{e}$ for $e \in S \cup T$.

One particularly simple class of rigidity circuits consists of the wheels. A wheel is a graph consisting of a cycle (the rim) and one additional vertex (the hub) with edges to all the rim vertices (the spokes). With this terminology established, we can state Martin's main theorem.

TheOREm 2.4. The polynomials $D_{W}$ for $W$ a wheel generate $I\left(\mathscr{S}_{n}\right)$. In fact, they form a Gröbner basis for this ideal, with respect to the graded lexicographic term order on the obvious lexicographic ordering of the variables $m_{i j}$. Moreover, the initial ideal $\operatorname{in}\left(I\left(\mathscr{S}_{n}\right)\right)$, and hence also I $\left(\mathscr{S}_{n}\right)$ itself, is Cohen-Macaulay, of dimension $2 n-3$ and degree

$$
M_{2 n-4}=(2 n-5)(2 n-7) \cdots 3 \cdot 1 .
$$

Let us say just a few words about the proof of this theorem, which involves a beautiful interplay of commutative algebra and combinatorics. By Theorem 2.3, the initial term of $D_{W}$ is a square-free "tree monomial" $m_{T}=\prod_{e \in T} m_{e}$, for some tree. Martin proves first that for wheels, the initial terms belong, not to arbitrary trees, but to trees which are paths, of the following special form. 
Definition 2.5. A Martin path in the graph $K_{n}$ on vertices $\{1, \ldots, n\}$ is a path $Q=(x, v, \ldots, w, y)$ such that (1) $x$ and $y$ are the two largest vertices of $Q$, and (2) assuming without loss of generality that $x<y$, then $v<w$.

Now the initial ideal $\operatorname{in}\left(D_{W}\right)$ of the ideal generated by wheel polynomials $D_{W}$ is the square-free monomial ideal generated by monomials $M_{Q}$ for $Q$ a Martin path. Hence

$$
R_{\Delta}=\mathbb{C}[\mathbf{m}] / \operatorname{in}\left(D_{W}\right)
$$

is the Stanley-Reisner ring of the simplicial complex $\Delta$ on the edge set of $K_{n}$, whose faces are those subgraphs $H \subseteq K_{n}$ that contain no Martin path. Martin proves next that this simplicial complex has the most optimal properties one could desire.

Proposition 2.6. Every maximal subgraph of $K_{n}$ containing no Martin paththat is, every facet of the simplicial complex $\Delta-$ has $2 n-3$ edges. The number of these facets is $M_{2 n-4}$. Moreover the complex $\Delta$ is shellable.

Shellability is a combinatorial property of a simplicial complex which implies in particular that it is Cohen-Macaulay, that is, the link of each face has only one nonzero reduced homology group. By a theorem of Hochster (see [Stanley 1996]), the latter property is equivalent to the Stanley-Reisner ring being CohenMacaulay. So Proposition 2.6 shows that the ideal

$$
J=\operatorname{in}\left(D_{W}: \text { all wheels } W\right)
$$

is Cohen-Macaulay, of dimension $2 n-3$ and degree $M_{2 n-4}$.

Finally, Martin uses a geometric argument to give a lower bound on the degree of the slope variety $\mathscr{S}_{n}$.

Proposition 2.7. The slope variety $\mathscr{S}_{n}$ has dimension $2 n-3$ and degree at least $M_{2 n-4}$.

Let us see where the above results leave us. We have two ideals, $J=\operatorname{in}\left(D_{W}\right)$, and $I=\operatorname{in} I\left(\mathscr{S}_{n}\right)$, and from the facts established so far we have:

(i) $J \subseteq I$,

(ii) $J$ is unmixed (since it is Cohen-Macaulay),

(iii) $\operatorname{dim} J=\operatorname{dim} I$,

(iv) $\operatorname{deg} J \leq \operatorname{deg} I$.

Together, these imply $J=I$, and Theorem 2.4 follows.

To close, I'll mention a striking combinatorial fact, which Martin left as a conjecture at the end of his thesis, but has since proved. The number $M_{2 n-4}$ is the number of matchings on $2 n-4$ vertices, that is, graphs in which every vertex is the endpoint of exactly one edge. The Hilbert series of the slope variety may be written

$$
\frac{h_{n}(q)}{(1-q)^{2 n-3}}
$$


where $h_{n}(q)$ is a polynomial with positive integer coefficients (because the ring is Cohen-Macaulay) and $h_{n}(1)=M_{2 n-4}$. Hence

$$
h_{n}(q)=a_{0}+a_{1} q+a_{2} q_{2}+\cdots
$$

is a $q$-analog of the number of matchings $M_{2 n-4}$. It turns out that it coincides with a combinatorial $q$-analog studied long ago by Kreweras and Poupard [1978].

THEOREM 2.8. The coefficient $a_{l}$ in the polynomial $h_{n}(q)$ is the number of matchings on the integers $\{1, \ldots, 2 n-4\}$ with $l$ long edges, where an edge $i, j$ is long if $|i-j| \neq 1$.

\title{
Appendix: Hilbert Schemes of Points in the Plane
}

\author{
By EzRA MilleR
}

Consider the polynomial ring $\mathbb{C}[x, y]$ in two variables over the complex numbers. As a set, the Hilbert scheme $H_{n}=\operatorname{Hilb}^{n}\left(\mathbb{C}^{2}\right)$ of $n$ points in the plane consists of those ideals $I \subseteq \mathbb{C}[x, y]$ such that the quotient $\mathbb{C}[x, y] / I$ has dimension $n$ as a vector space over $\mathbb{C}$. This appendix provides some background on how this set can be considered naturally as a smooth algebraic variety of dimension $2 n$. The goal is to orient the reader rather than to give a complete introduction. Therefore some details are omitted from the exposition to make the intuition more clear (and short). The material here, which is based loosely on the introductory parts of [Haiman 1998], reflects what was presented at the help session for Haiman's lectures; in particular, the Questions were all asked by participants at the help session.

To begin, let's get a feeling for what an ideal $I$ of colength $n$ can look like. If $P_{1}, \ldots, P_{n} \in \mathbb{C}^{2}$ are distinct (reduced) points, for example, then the ideal of functions vanishing on these $n$ points has colength $n$. This is because the ring of functions on $n$ points has a vector space basis $\left\{f_{1}, \ldots, f_{n}\right\}$ in which $f_{i}\left(P_{j}\right)=0$ unless $i=j$, and $f_{i}\left(P_{i}\right)=1$. Ideals of the form $I\left(P_{1}, \ldots, P_{n}\right)$ are called generic colength $n$ ideals.

At the opposite end of the spectrum, $I$ could be an ideal whose (reduced) zero set consists of only one point $P \in \mathbb{C}^{2}$. In this case, $\mathbb{C}[x, y] / I$ is a local ring with lots of nilpotent elements. In geometric terms, this means that $P$ carries a nonreduced scheme structure. Such a nonreduced scheme structure on $P$ is far from unique; in other words, there are many length $n$ local rings $\mathbb{C}[x, y] / I$ supported at $P$. In fact, they come in an $(n-1)$-dimensional family.

Among the ideals supported at single points, the monomial ideals are the most special. These ideals have the form $I=\left\langle x^{a_{1}} y^{b_{1}}, \ldots, x^{a_{m}} y^{b_{m}}\right\rangle$ for some nonnegative integers $a_{1}, b_{1}, \ldots, a_{m}, b_{m}$, and are supported at $(0,0) \in \mathbb{C}^{2}$. Note that if $x^{h} y^{k}$ is a monomial outside of $I$ and $x^{h^{\prime}} y^{k^{\prime}}$ is a monomial dividing $x^{h} y^{k}$ (so $h^{\prime} \leq h$ and $k^{\prime} \leq k$ ), then $x^{h^{\prime}} y^{k^{\prime}}$ also lies outside of $I$. This makes it convenient to draw the monomials outside of $I$ as the boxes "under a staircase". 
EXAmple A.1. For the ideal $I=\left\langle x^{2}, x y, y^{3}\right\rangle$ of colength $n=4$, the diagram of boxes under the staircase is L-shaped:

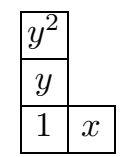

Note that the monomial $x^{2}$ would be the first box after the bottom row, while $x y$ would nestle in the nook of the ' $\mathrm{L}$ ', and $y^{3}$ would lie atop the first column. Thus the minimal generators of $I$ specify where to draw the staircase.

If the diagram of monomials outside $I$ has $\lambda_{i}$ boxes in row $i$ under the staircase, then $\sum_{i} \lambda_{i}=n$ is by definition a partition $\lambda$ of $n$, and we write $I=I_{\lambda}$.

ExAmple A.2. In Example A.1, there are 2 boxes in row 0, and 1 box in each of rows 1 and 2 , yielding the partition $2+1+1=4$ of $n=4$. Thus the ideal is $I=I_{2+1+1}$.

In full generality, the quotient $\mathbb{C}[x, y] / I$ is a product of local rings with maximal ideals corresponding to a finite set $P_{1}, \ldots, P_{r}$ of distinct points in $\mathbb{C}^{2}$, with the lengths $\ell_{1}, \ldots, \ell_{r}$ of these local rings satisfying satisfying $\ell_{1}+\cdots+\ell_{r}=n$ (do not confuse this partition of $n$ with the partitions obtained from monomial ideals, where $r=1$ ). When $r=n$ it must be that $\ell_{i}=1$ for all $i$, so the ideal $I$ is generic.

QUESTION 1. Is there some transformation of the plane so that every colength $n$ ideal has a basis of monomials?

ANSwER 1. This question can be interpreted in two different ways, because the word "basis" has multiple meanings. Thinking of "basis" as "generating set", the question asks if given $I$, there is a coordinate system for $\mathbb{C}^{2}$ in which $I$ is a monomial ideal. The answer is no, in general; for instance, if $\mathbb{C}[x, y] / I$ is not a local ring, then $I$ can't be a monomial ideal in any coordinates. The second meaning of "basis" is " $\mathbb{C}$-vector space basis". Even though $I$ itself may not be expressible in some coordinates as a monomial ideal, the quotient $\mathbb{C}[x, y] / I$ always has a $\mathbb{C}$-vector space basis of (images of) monomials. This observation will be crucial later on.

If all colength $n$ ideals were generic, then the set $H_{n}$ would be easy to describe, as follows. Every unordered list of $n$ distinct points in $\mathbb{C}^{2}$ corresponds to a set of $n$ ! points in $\left(\mathbb{C}^{2}\right)^{n}$, or alternatively to a single point in the quotient $S^{n} \mathbb{C}^{2}:=\left(\mathbb{C}^{2}\right)^{n} / S_{n}$ by the symmetric group. Of course, not every point of $S^{n} \mathbb{C}^{2}$ corresponds to an unordered list of distinct points; for that, one needs to remove the diagonals

$$
\left\{\left(P_{1}, \ldots, P_{n}\right) \in\left(\mathbb{C}^{2}\right)^{n} \mid P_{i}=P_{j}\right\}
$$

of $\left(\mathbb{C}^{2}\right)^{n}$ before quotienting by $S_{n}$. Since $S_{n}$ acts freely on the complement $\left(\left(\mathbb{C}^{2}\right)^{n}\right)^{\circ}$ of the diagonals $(\mathrm{A}-1)$, the complement $\left(S^{n} \mathbb{C}^{2}\right)^{\circ}$ of the diagonals in 
the quotient $S^{n} \mathbb{C}^{2}$ is smooth. Therefore, whatever variety structure we end up using, $H_{n}$ will contain an open smooth subvariety $\left(S^{n} \mathbb{C}^{2}\right)^{\circ}$ of dimension $2 n$ parametrizing generic ideals.

The variety structure on $H_{n}$ arises by identifying it as an algebraic subvariety of a more familiar variety: a grassmannian. Consider the vector subspace $V_{d}$ inside of $\mathbb{C}[x, y]$ spanned by the $\left(\begin{array}{c}d+2 \\ 2\end{array}\right)$ monomials of degree at most $d$.

Lemma A.3. Fix $d \geq n$. Given any colength $n$ ideal $I$, the image of $V_{d}$ spans the quotient $\mathbb{C}[x, y] / I$ as a vector space.

Proof. The $n$ monomials outside any initial ideal of $I$ span the quotient $\mathbb{C}[x, y] / I$, and these monomials must lie inside $V_{d}$.

The intersection $I \cap V_{d}$ is a vector subspace of codimension $n$. Thus $H_{n}$ is (as a set, at least) contained in the grassmannian $\operatorname{Gr}^{n}\left(V_{d}\right)$ of codimension $n$ subspaces of $V_{d}$.

Definition A.4. Given a partition $\lambda$ of $n$, write $U_{\lambda} \subset H_{n}$ for the set of ideals $I$ such that the monomials outside $I_{\lambda}$ map to a vector space basis for $\mathbb{C}[x, y] / I$.

The set of codimension $n$ subspaces $W \subset V_{d}$ for which the monomials outside $I_{\lambda}$ span $V_{d} / W$ constitutes a standard open affine subvariety of $\operatorname{Gr}^{n}\left(V_{d}\right)$, defined by the nonvanishing of the corresponding Plücker coordinate. This means that $W$ has a unique basis consisting of vectors of the form

$$
x^{r} y^{s}-\sum_{h k \in \lambda} c_{h k}^{r s} x^{h} y^{k} \quad \text { for } 0 \leq r+s \leq d .
$$

Here, we write $h k \in \lambda$ to mean $x^{h} y^{k} \notin I_{\lambda}$, so the box labeled $(h, k)$ lies under the staircase for $I_{\lambda}$. The affine open inside $\operatorname{Gr}^{n}\left(V_{d}\right)$ is actually a cell - namely, the variety whose coordinate ring is the polynomial ring in the coefficients $c_{h k}^{r s}$ from $(\mathrm{A}-2)$.

The intersection of each ideal $I \in U_{\lambda}$ with $V_{d}$ is a codimension $n$ subspace of $V_{d}$ spanned by vectors of the form $(\mathrm{A}-2)$, by definition of $U_{\lambda}$. Of course, if $W \subset V_{d}$ is to be expressible as the intersection of $V_{d}$ with some ideal $I$, the coefficients $c_{h k}^{r s}$ can't be chosen completely at will. Indeed, the fact that $I$ is an ideal imposes relations on the coefficients that say "multiplication by $x$ takes $x^{r} y^{s}$ to $x^{r+1} y^{s}$ and preserves $I$, and similarly for multiplication by $y$."

Explicitly, if $x^{r+1} y^{s} \in V_{d}$, then multiplying (A-2) by $x$ yields another polynomial $x^{r+1} y^{s}-\sum_{h k \in \lambda} c_{h k}^{r s} x^{h+1} y^{k}$ inside $I \cap V_{d}$. Some of the terms $x^{h+1} y^{k}$ no longer lie outside $I_{\lambda}$, so we have to expand them again using $(\mathrm{A}-2)$ to get

$$
x^{r+1} y^{s}-\left(\sum_{h+1, k \in \lambda} c_{h k}^{r s} x^{h+1} y^{k}+\sum_{h+1, k \notin \lambda} c_{h k}^{r s} \sum_{h^{\prime} k^{\prime} \in \lambda} c_{h^{\prime} k^{\prime}}^{h+1, k} x^{h^{\prime}} y^{k^{\prime}}\right) \in I .
$$

Equating the coefficients on $x^{h} y^{k}$ in $(\mathrm{A}-3)$ to those in

$$
x^{r+1} y^{s}-\sum_{h k \in \lambda} c_{h k}^{r+1, s} x^{h} y^{k}
$$


from (A-2) yields relations in the polynomial ring $\mathbb{C}\left[\left\{c_{h k}^{r s}\right\}\right]$. These relations, taken along with their counterparts that result by switching the roles of $x$ and $y$, cut out $U_{\lambda}$. Though we have yet to see that these relations generate a radical ideal, we can at least conclude that $U_{\lambda}$ is an algebraic subset of an open cell in the grassmannian.

Theorem A.5. Fix $d \geq n+1$. The affine varieties $U_{\lambda}$ cover the subset $H_{n} \subset$ $\operatorname{Gr}^{n}\left(V_{d}\right)$, thereby endowing $H_{n}$ with the structure of quasiprojective algebraic variety.

Proof. The sets $U_{\lambda}$ cover $H_{n}$ by Lemma A.3, and each set $U_{\lambda}$ is locally closed in $\operatorname{Gr}^{n}\left(V_{d}\right)$ by the discussion above.

In summary: $H_{n}$ is a quasiprojective variety because it is locally obtained by the intersection of a Zariski open condition (certain monomials span mod $I$ ) and a Zariski closed condition ( $W \subset V_{d}$ is closed under multiplication by $x$ and $y$ ).

Theorem A.5 does not claim that the variety structure is independent of $d$, although it is true (and important), and can be deduced using smoothness of $H_{n}$ (Theorem A.14) along with the fact that projection $V_{d+1} \rightarrow V_{d}$ maps $H_{n}$ to itself by sending $I \cap V_{d+1} \mapsto I \cap V_{d}$. Had we allowed $d=n$, however, where Proposition A.12 can fail, the variety structure might be different. In any case, fix $d \geq n+1$ in the forthcoming discussion.

Having endowed $H_{n}$ with an algebraic variety structure, let us explore its properties.

Lemma A.6. Every point $I \in H_{n}$ is connected to a monomial ideal by a rational curve.

PROof. Choosing a term order and taking a Gröbner basis of $I$ yields a family of ideals parametrized by the coordinate variable $t$ on the affine line. When $t=1$ we get $I$ back, and when $t=0$ we get the initial ideal of $I$, which is a monomial ideal.

This proof is stated somewhat vaguely, but can be made quite precise using the notion of flat family and the fact that Gröbner degenerations are flat families over the affine line [Eisenbud 1995, Proposition 15.17]. Here is an example, for more concrete intuition.

EXAmple A.7. Suppose $I=\left\langle x^{2}, x y+\sqrt{2} x, y^{3}-2 y\right\rangle$, and consider the ideal

$$
I_{t}=\left\langle x^{2}, x y+\sqrt{2} t x, y^{3}-2 t y\right\rangle \subset \mathbb{C}[x, y][t] .
$$

This new ideal should be thought of as a family of ideals in $\mathbb{C}[x, y]$, parametrized by the coordinate $t$. The ideal at $\alpha \in \mathbb{C}$ is obtained by setting $t=\alpha$ in the generators for $I_{t}$. Every one of these ideals has colength 4, because they all have the ideal $\left\langle x^{2}, x y, y^{3}\right\rangle$ from Example A.1 as an initial ideal. It follows that this family of ideals (or better yet, the family $\mathbb{C}[x, y][t] / I_{t}$ of quotients) is flat over $\mathbb{C}[t]$. 
Lemma A.6 allows us to conclude the following:

Proposition A.8. The Hilbert scheme $H_{n}$ is connected.

Question 2. Lemma A.6 only says that every ideal connects to some monomial ideal. How do you know that you can get from one monomial ideal to another?

ANSWER 2. They're all connected to generic ideals:

Lemma A.9. For every partition $\lambda$ of $n$, the point $I_{\lambda} \in H_{n}$ lies in the closure of the generic locus $\left(S^{n} \mathbb{C}^{2}\right)^{\circ}$.

Proof. Consider the set of exponent vectors $(h, k)$ on monomials $x^{h} y^{k}$ outside $I$ as a subset of $\mathbb{Z}^{2} \subset \mathbb{C}^{2}$. These exponent vectors constitute a collection of $n$ points in $\mathbb{C}^{2}$. The colength $n$ ideal of these points is called the distraction $I_{\lambda}^{\prime}$ of $I_{\lambda}$. If $I_{\lambda}=\left\langle x^{a_{1}} y^{b_{1}}, \ldots, x^{a_{m}} y^{b_{m}}\right\rangle$, then $I_{\lambda}^{\prime}=\left\langle f_{1}, \ldots, f_{m}\right\rangle$, where

$$
f_{i}=x(x-1)(x-2) \cdots\left(x-a_{i}+2\right)\left(x-a_{i}+1\right) y(y-1) \cdots\left(y-b_{i}+1\right) .
$$

Indeed, this ideal has colength $n$ because every term of $f_{i}$ divides its leading term $x^{a_{i}} y^{b_{i}}$, forcing $I_{\lambda}$ to be the unique initial ideal of $\left\langle f_{1}, \ldots, f_{m}\right\rangle$; and each polynomial $f_{i}$ clearly vanishes on the exponent set of $I_{\lambda}$, so each $f_{i}$ lies in $I_{\lambda}^{\prime}$.

Example A.10. The distraction of $I_{2+1+1}=\left\langle x^{2}, x y, y^{3}\right\rangle$ is the ideal

$$
I_{2+1+1}^{\prime}=\langle x(x-1), x y, y(y-1)(y-2)\rangle .
$$

The zero set of every generator of the distraction is a union of lines, namely integer translates of one of the two coordinate axes in $\mathbb{C}^{2}$. The zero set of our ideal $I_{2+1+1}^{\prime}$ is

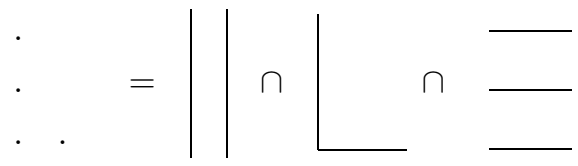

The groups of lines on the right hand side are the zero sets of $x(x-1), x y$, and $y(y-1)(y-2)$, respectively.

Remark A.11. Proposition A.8 holds for Hilbert schemes of $n$ points in $\mathbb{C}^{m}$ even when $m$ is arbitrary, with the same proof. Hartshorne's connectedness theorem [Hartshorne 1966] says that it holds for certain more general Hilbert schemes, under the $\mathbb{Z}$-grading. However, the result does not extend to Hilbert schemes under arbitrary gradings [Haiman and Sturmfels 2002; Santos 2002].

Proposition A.12. For each $\lambda$, the local ring of $H_{n} \subset \operatorname{Gr}^{n}\left(V_{d}\right)$ at $I_{\lambda}$ has embedding dimension at most $2 n$; that is, the maximal ideal $\mathfrak{m}_{I_{\lambda}}$ satisfies

$$
\operatorname{dim}_{\mathbb{C}}\left(\mathfrak{m}_{I_{\lambda}} / \mathfrak{m}_{I_{\lambda}}^{2}\right) \leq 2 n
$$


Proof. Identify each variable $c_{h k}^{r s}$ with an arrow pointing from the box $h k \in \lambda$ to the box $r s \notin \lambda$ (see Example A.13). Allow arrows starting in boxes with $h<0$ or $k<0$, but set them equal to zero. The arrows lie inside - and in fact generate - the maximal ideal $\mathfrak{m}_{I_{\lambda}}$ at the point $I_{\lambda} \in H_{n}$. As each term in the double sum in (A-3) has two $c$ 's in it, the double sum lies inside $\mathfrak{m}_{I_{\lambda}}^{2}$. Moving both the tail and head of any given arrow one box to the right therefore does not change the arrow's residue class modulo $\mathfrak{m}_{I_{\lambda}}^{2}$, as long as the tail of the original arrow does not end up past the last box in a row of $\lambda$, and the head of the arrow does not end up on a monomial of degree strictly larger than $d$. Switching the roles of $x$ and $y$, we conclude that an arrow's residue class $\bmod \mathfrak{m}_{I_{\lambda}}^{2}$ is unchanged by moving vertically or horizontally, as long as the tail stays under the staircase, while the head stays above it (but still inside the set of monomials of degree at most $d$ ). This analysis includes the case where the tail of the arrow crosses either axis, in which case the arrow is zero.

Using the fact that $d \geq n+1$ in Theorem A.5 to pass the head through corners $(h+1, k+1)$ for $(h, k) \in \lambda$, every arrow can be moved horizontally and vertically until either

(i) the tail crosses an axis; or

(ii) there is a box $h k \in \lambda$ such that the tail lies just inside row $k$ of $\lambda$ while the head lies just above column $h$ outside $\lambda$; or

(iii) there is a box $h k \in \lambda$ such that the tail lies just under the top of column $h$ in $\lambda$ while the head lies in the first box to the right outside row $k$ of $\lambda$.

Arrows of the first sort do not contribute at all to $\mathfrak{m}_{I_{\lambda}} / \mathfrak{m}_{I_{\lambda}}^{2}$. On the other hand, there are exactly $n$ northwest-pointing arrows of the second sort, and exactly $n$ southeast-pointing arrows of the third sort. Therefore $\mathfrak{m}_{I_{\lambda}} / \mathfrak{m}_{I_{\lambda}}^{2}$ has dimension at most $2 n$.

EXAMPLE A.13. All three figures below depict the same partition $\lambda: 8+8+5+$ $3+3+3+3+2=35$. In the left figure, the middle of the five arrows represents $c_{31}^{54} \in \mathfrak{m}_{I_{\lambda}}$. As in the proof of Proposition A.12, all of the arrows in the left figure are equal modulo $\mathfrak{m}_{I_{\lambda}}^{2}$. Since the bottom one is manifestly zero as in item (i) from the proof of Proposition A.12, all of the arrows in the left figure represent zero in $\mathfrak{m}_{I_{\lambda}} / \mathfrak{m}_{I_{\lambda}}^{2}$.
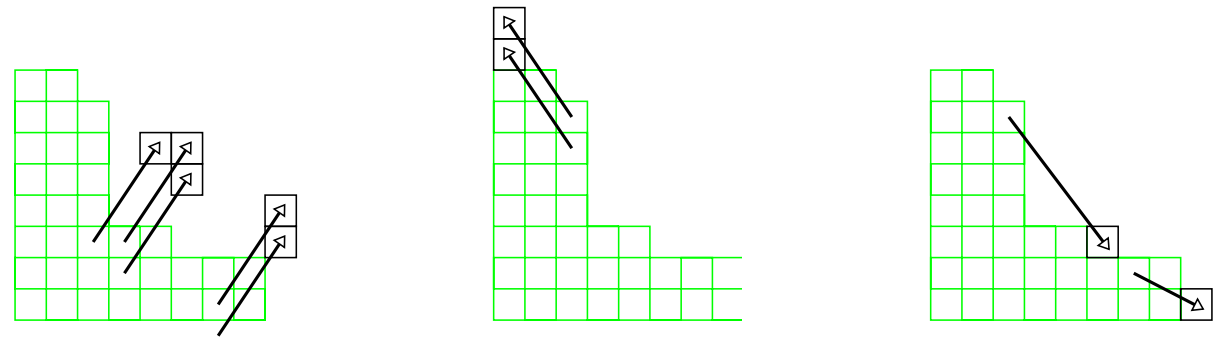
The two arrows in the middle figure are equal, and the bottom one $c_{25}^{08}$ provides an example of a regular parameter in $\mathfrak{m}_{I_{\lambda}}$ as in (ii). Finally, the two arrows in the rightmost figure represent unequal regular parameters as in (iii).

Now we finally have enough prerequisites to prove the main result.

THEOREM A.14. The Hilbert scheme $H_{n}$ is a smooth and irreducible subvariety of dimension $2 n$ inside $\mathrm{Gr}^{n}\left(V_{d}\right)$ for $d \geq n+1$.

Proof. Since the intersection of two irreducible components would be contained in the singular locus of $H_{n}$, it is enough by Proposition A.8 to prove smoothness. Lemma A.9 implies that the dimension of the local ring of $H_{n}$ at any monomial ideal $I_{\lambda}$ is at least $2 n$, because the generic locus has dimension $2 n$. On the other hand, Proposition A.12 shows that the maximal ideal of that local ring can be generated by $2 n$ polynomials. Therefore $H_{n}$ is regular in a neighborhood of any point $I_{\lambda}$.

The two-dimensional torus acting on $\mathbb{C}^{2}$ by scaling the coordinates has an induced action on $H_{n}$. Under this action, Lemma A.6 and its proof say that every orbit on $H_{n}$ contains a monomial ideal (= torus-fixed point) in its closure. By general principles, the singular locus of $H_{n}$ must be torus-fixed (though not necessarily pointwise, of course) and closed. Since every torus orbit on $H_{n}$ contains a smooth point of $H_{n}$ in its closure, the singular locus must be empty.

The proof of Theorem A.14 used the fact that Gröbner degenerations are accomplished by taking limits of one-parameter torus actions on $H_{n}$. In plain language, this means simply that if appropriate powers of $t$ are used in the equations defining the family $I_{t}$, the variable $t$ can be thought of as a coordinate on $\mathbb{C}^{*}$ for nonzero values of $t$.

Remark A.15. Theorem A.14 fails for Hilbert schemes $\operatorname{Hilb}^{n}\left(\mathbb{C}^{m}\right)$ of points in spaces of dimension $m \geq 3$, as proved by Iarrobino [Iarrobino 1972]. If it were irreducible, then $\operatorname{Hilb}^{n}\left(\mathbb{C}^{m}\right)$ would have dimension $m n$, the dimension of the open subset of configurations of $n$ distinct points. But Iarrobino constructed a dimension $e$ family of ideals of colength $n$ in the polynomial ring, where $e$ is proportional to $n^{(2-2 / m)}$. It follows that $\operatorname{Hilb}^{n}\left(\mathbb{C}^{m}\right)$ is in fact reducible for $m \geq 3$ and $n$ sufficiently large. On the other hand, $\operatorname{Hilb}^{n}\left(\mathbb{C}^{m}\right)$ is connected by reasoning as in the case $n=2$ (Lemma A.6 and Lemma A.9).

Question 3. Is the open set $U_{\lambda} \subset H_{n}$ the locus of colength $n$ ideals having $I_{\lambda}$ as an initial ideal?

Answer 3. When $\lambda$ is the partition $1+\cdots+1=n$, then yes. Otherwise, no, since the set of such ideals has dimension strictly less than $2 n$. However, the locus in $H_{n}$ of ideals having initial ideal $I_{\lambda}$ is cell - that is, isomorphic to $\mathbb{C}^{m}$ for some $m$. Lemma A.6 can be interpreted as saying that $H_{n}$ is the disjoint union of these cells. This is the Biatynicki-Birula decomposition of $H_{n}$ [Białynicki-Birula 1976; Ellingsrud and Strømme 1987]. It exists essentially because $H_{n}$ has an 
action of the torus $\left(\mathbb{C}^{*}\right)^{2}$ with isolated fixed points. Knowledge of the BiałynickiBirula decomposition allows one to compute the cohomology ring of $H_{n}$, which was the purpose of [Ellingsrud and Strømme 1987].

\section{References}

[Allen 1993] E. E. Allen, "A conjecture of Procesi and a new basis for the decomposition of the graded left regular representation of $S_{n}$ ", Adv. Math. 100:2 (1993), 262-292.

[Białynicki-Birula 1976] A. Białynicki-Birula, "Some properties of the decompositions of algebraic varieties determined by actions of a torus", Bull. Acad. Polon. Sci. Sér. Sci. Math. Astronom. Phys. 24:9 (1976), 667-674.

[Chevalley 1955] C. Chevalley, "Invariants of finite groups generated by reflections", Amer. J. Math. 77 (1955), 778-782.

[Derksen 1999] H. Derksen, "Computation of invariants for reductive groups", Adv. Math. 141:2 (1999), 366-384.

[Eisenbud 1995] D. Eisenbud, Commutative algebra, with a view toward algebraic geometry, Graduate Texts in Mathematics 150, Springer, New York, 1995.

[Ellingsrud and Strømme 1987] G. Ellingsrud and S. A. Strømme, "On the homology of the Hilbert scheme of points in the plane", Invent. Math. 87:2 (1987), 343-352.

[Fogarty 1968] J. Fogarty, "Algebraic families on an algebraic surface", Amer. J. Math 90 (1968), 511-521.

[Garsia and Haglund 2001] A. M. Garsia and J. Haglund, "A positivity result in the theory of Macdonald polynomials", Proc. Natl. Acad. Sci. USA 98:8 (2001), 43134316.

[Garsia and Haglund 2002] A. M. Garsia and J. Haglund, "A proof of the $q, t$-Catalan positivity conjecture", Discrete Math. 256:3 (2002), 677-717.

[Gordon 2003] I. Gordon, "On the quotient ring by diagonal invariants", Invent. Math. 153:3 (2003), 503-518.

[Haiman 1994] M. D. Haiman, "Conjectures on the quotient ring by diagonal invariants", J. Algebraic Combin. 3:1 (1994), 17-76.

[Haiman 1998] M. Haiman, " $t, q$-Catalan numbers and the Hilbert scheme", Discrete Math. 193:1-3 (1998), 201-224.

[Haiman 2001] M. Haiman, "Hilbert schemes, polygraphs and the Macdonald positivity conjecture", J. Amer. Math. Soc. 14:4 (2001), 941-1006.

[Haiman 2002] M. Haiman, "Vanishing theorems and character formulas for the Hilbert scheme of points in the plane", Invent. Math. 149:2 (2002), 371-407.

[Haiman and Sturmfels 2002] M. Haiman and B. Sturmfels, "Multigraded Hilbert schemes", 2002. Available at arXiv:math.AG/0201271.

[Hartshorne 1966] R. Hartshorne, "Connectedness of the Hilbert scheme", Inst. Hautes Études Sci. Publ. Math. 29 (1966), 5-48.

[Hochster and Eagon 1971] M. Hochster and J. A. Eagon, "Cohen-Macaulay rings, invariant theory, and the generic perfection of determinantal loci", Amer. J. Math. 93 (1971), 1020-1058. 
[Iarrobino 1972] A. Iarrobino, "Reducibility of the families of 0-dimensional schemes on a variety", Invent. Math. 15 (1972), 72-77.

[Kreweras and Poupard 1978] G. Kreweras and Y. Poupard, "Sur les partitions en paires d'un ensemble fini totalement ordonné", Publ. Inst. Statist. Univ. Paris 23:12 (1978), 57-74.

[Martin 2003a] J. L. Martin, "Geometry of graph varieties", Trans. Amer. Math. Soc. 355:10 (2003), 4151-4169.

[Martin 2003b] J. L. Martin, "The slopes determined by $n$ points in the plane", 2003. Available at arXiv:math.AG/0302106.

[Orlik and Terao 1992] P. Orlik and H. Terao, Arrangements of hyperplanes, Grundlehren Math. Wiss. 300, Springer, Berlin, 1992.

[Santos 2002] F. Santos, "Non-connected toric Hilbert schemes", 2002. Available at arXiv:math.CO/0204044.

[Shephard and Todd 1954] G. C. Shephard and J. A. Todd, "Finite unitary reflection groups", Canadian J. Math. 6 (1954), 274-304.

[Stanley 1996] R. P. Stanley, Combinatorics and commutative algebra, Second ed., Progress in Mathematics 41, Birkhäuser, Boston, MA, 1996.

[Steinberg 1960] R. Steinberg, "Invariants of finite reflection groups", Canad. J. Math. 12 (1960), 616-618.

[Steinberg 1964] R. Steinberg, "Differential equations invariant under finite reflection groups", Trans. Amer. Math. Soc. 112 (1964), 392-400.

[Watanabe 1974] K. Watanabe, "Certain invariant subrings are Gorenstein, I and II", Osaka J. Math. 11 (1974), 1-8, 379-388.

[Weyl 1939] H. Weyl, The classical groups: their invariants and representations, Princeton University Press, Princeton, 1939.

\author{
MARK HAIMAN \\ DePARTMENT OF MATHEMATICS \\ UNIVERSITY OF CALIFORNIA \\ 970 EVANS HALL \\ BERKELEy, CA 94720-3840 \\ United States \\ mhaiman@math.berkeley.edu
}

Ezra Miller

School of Mathematics

UNIVERSITY OF MINNESOTA

127 VINCENT HALL

206 Church Street, SE

MiNnEAPOLIS, MN 55455

United States

ezra@math.umn.edu 